\title{
Magnetic resonance imaging features of vaginal endometriosis
}

\author{
Valeria Fiaschetti, Valentina Cama, Laura Greco, Maria Fornari, \\ Giuseppe Sorrenti, Giovanni Simonetti
}

\begin{abstract}
Introduction: Deeply infiltrating endometriosis (DIE) is defined by the presence of endometrial implants penetrating under the peritoneal surface or under the wall of the pelvic organs to a depth of at least $5 \mathrm{~mm}$. This case report describes a case of DIE involving vaginal wall, periurethral tissue, torus uterinus and sigmoid wall. Case Report: A 46-year-old female was presented with pelvic pain and deep dyspareunia which started six years ago, after a spontaneous interruption of pregnancy and uterine curettage. The pelvic magnetic resonance imaging (MRI) showed multiple DIE lesions localized in the vaginal wall mainly in the lower third of the vagina, between the anterior vaginal wall and the urethra, on the contour of the urethra and between the torus uterinus and the sigmoid wall. After MRI, laparoscopic biopsies confirmed the DIE lesions. Conclusion: According to the
\end{abstract}

Valeria Fiaschetti ${ }^{1}$, Valentina Cama ${ }^{2}$, Laura Greco ${ }^{2}$, Maria Fornari $^{2}$, Giuseppe Sorrenti ${ }^{3}$, Giovanni Simonetti ${ }^{4}$

Affiliations: ${ }^{1}$ Research, Department of Diagnostic Imaging, Molecular Imaging, Interventional Radiology and Radiation Therapy. University Hospital Tor Vergata, Rome, Italy; ${ }^{2}$ Resident, Department of Diagnostic Imaging, Molecular Imaging, Interventional Radiology and Radiation Therapy. University Hospital Tor Vergata, Rome, Italy; ${ }^{3} \mathrm{MD}$, Department of Gynecology and Obstetrics, University Hospital Tor Vergata, Rome, Italy; ${ }^{4}$ Professor, Department of Diagnostic Imaging, Molecular Imaging, Interventional Radiology and Radiation Therapy. University Hospital Tor Vergata, Rome, Italy

Corresponding Author: Valentina Cama, Viale Oxford 81, Rome, Italy 00133; Ph: +390620902400; Fax: +390620902404; Email: valentinacama@gmail.com

Received: 22 November 2012

Accepted: 12 January 2013

Published: 01 August 2013 implantion theory, we assume that in our case the vaginal endometriosis was the result of implantation of endometrial glands into the vaginal tearing during the surgical procedure of curettage. In the current literature no author has so far described a diffuse involvement of the vaginal wall in the presence of uterine curettage after interruption of pregnancy. In our case, the anterior (peri-urethral tissue), middle (vaginal wall) and posterior (torus uterinus and bowel wall) compartments are involved. We can presume that the vaginal DIE is an early lesion, while the others lesions arise from vaginal walls by a contiguous extension. On MRI with endovaginal gel these lesions were more evident rather than without gel. MRI with gel allows us to give essential preoperative mapping for the surgical removal of the DIE lesions.

Keywords: Vaginal endometriosis, Deeply infiltrating endometriosis (DIE), Endovaginal gel

$$
* * * * * * * * *
$$

Fiaschetti V, Cama V, Greco L, Fornari M, Sorrenti G, Simonetti G. Magnetic resonance imaging features of vaginal endometriosis. International Journal of Case Reports and Images 2013;4(8):431-435.

$$
* * * * * * * *
$$

doi:10.5348/ijcri-2013-08-349-CR-8

\section{INTRODUCTION}

Endometriosis is a chronic gynecologic disorder affecting women in reproductive age; in the general population the prevalence of endometriosis is $10 \%$ [1]. It is characterized by the presence of functional 
endometrial glands and stroma outside the uterine cavity. The most common localizations of endometriosis are the ovaries and the pelvic peritoneum, followed by deep lesions of the pelvic subperitoneal space. Deeply infiltrating endometriosis (DIE) is a specific entity, histologically defined by the presence of endometrial implants penetrating under the peritoneal surface or under the wall of the pelvic organs to a depth of at least $5 \mathrm{~mm}$ [2]. It involves more frequently fibromuscolar structures such as uterosacral legaments and rectovaginal septum (RVS) (69.2\% of cases), rarely the vagina (14.5\%) [3]. This case report describes a case of DIE involving vaginal wall, periurethral tissue and torus uterinus with involvement of the sigmoid wall.

\section{CASE REPORT}

We reported a case of a 46-year-old female. Her clinical history consisted of exacerbated pelvic pain during her menstrual cycle and deep dyspareunia which started six years ago, after spontaneous interruption of pregnancy and uterine curettage.

The clinical examination detected painful thickening of the vagina but the ultrasonography did not report abnormal findings except for bilateral ovarian cystis and multiple small uterin fibroids. Vaginal bleeding was observed after introduction of the speculum.

The patient was referred by the Department of Gynecology and Obstetrics for clinical suspicion of DIE which was based on the detection of painful thickening of the vagina on physical examination.

We performed a pelvic magnetic resonance imaging (MRI) with a $1.5 \mathrm{~T}$ scanner (Achieva, Philips medical systems, BEST, Netherlands) using a pelvic phasedarray coil. We used peristaltic inhibitors (10 $\mathrm{mg}$ of hyoscine-N-butylbromide, buscopan) diluted in $10 \mathrm{~mL}$ saline to reduce gastrointestinal tract peristaltis.

The imaging protocol included a T1-weighted (W) turbo spin-echo (TSE), a T2-W TSE, a T1-W TSE fatsaturation, performed in the transverse plane (perpendicular to axis of cervix). A T2-W TSE sequences and a T1-W TSE fat-saturation were performed on sagittal plane. The transverse and sagittal T2-W TSE and the transverse and sagittal T1-W TSE fat-saturation sequences were performed before and after the introduction of ultrasonographic gel $(100 \mathrm{~mL})$ into the vaginal canal. No intravenous contrast medium was administered.

The MRI showed a hypointense concentric thickening of the vaginal wall on T2-W sequences. The thickening was mainly evident in the lower third of the vagina, where it caused pseudostenosis. On the sagittal, T2-W sequence with endovaginal gel this finding was more evident than that without the use of the gel (Figure 1A-B). On T2-W sequence between anterior vaginal wall and urethra, above the perineal body, a hyperintense focus was revealed. It was more evident on the sequence with gel (Figure 1B). On the T1-fat, saturation sequence showed a hyperintense signal indicating bloody content. Urethral involvement was suspected for hypointense irregular spiculated mass-like thickening on the contour of the urethra involving the anterior vaginal wall (Figure 2). Thin hyperintense lines were revealed in the posterior vaginal wall, clearly visible in the $\mathrm{T} 1$ fat-saturation sequence after the introduction of gel into the vagina indicating bloody content (Figure 3). The T2-W sequence showed thin hypointense lines located between the torus uterinus and the sigmoid related to retractile adhesions (Figure 4). After MRI, patient underwent to multiple biopsies to obtain a definitive diagnose. Histopathology tests confirmed islands of endometrial tissues and stroma in the vaginal wall, periurethral tissue and torus uterinus.
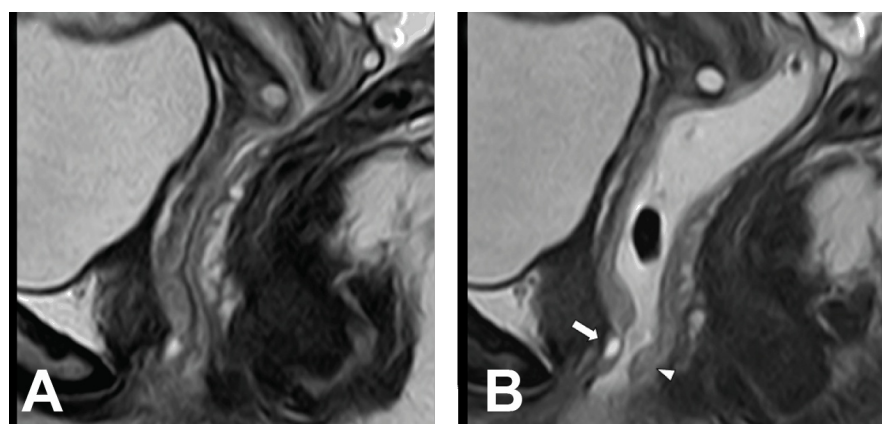

Figure 1: (A, B) Sagittal T2-weighted sequences shows a diffuse thickening vaginal wall and a hyperintense oval lesion localized between the anterior vaginal wall and the urethra, above the perineal body. (A) It is poorly detectable without gel The vaginal pre-orificial pseudostenosis was not appreciable on the image without gel. (B) The image after gel allows to better evaluate the thickening of the vaginal wall, mainly evident in the lower third where it caused pseudostenosis (arrowhead) and the oval focus (arrow).

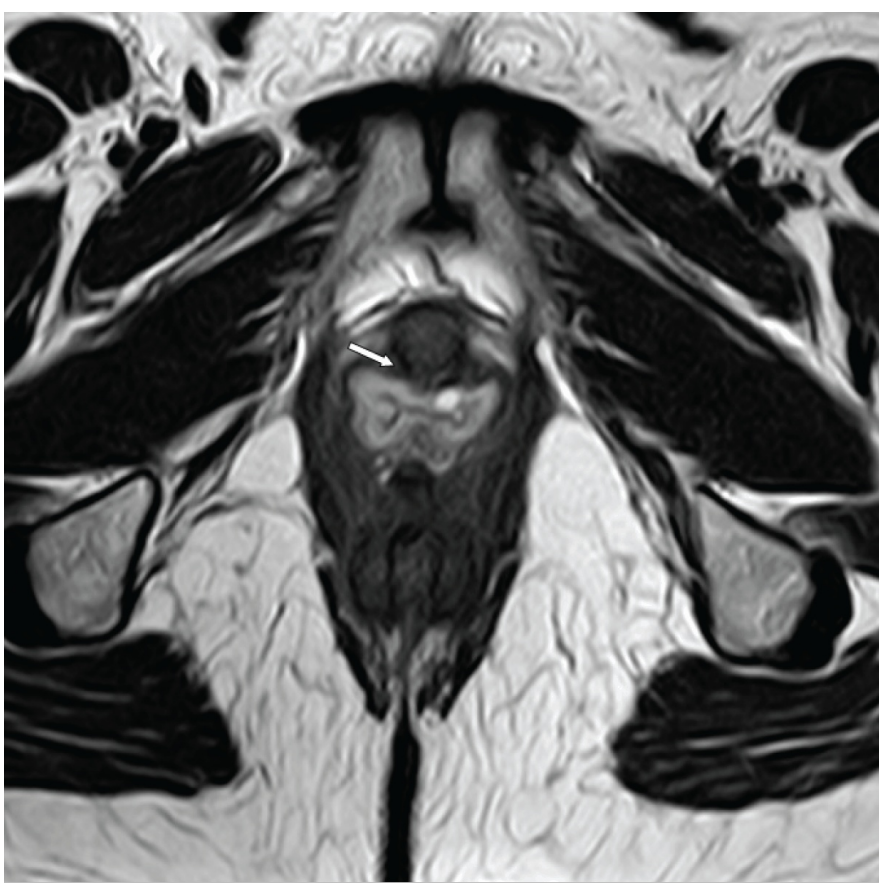

Figure 2: Axial T2-weighted sequence shows an hypointense irregular spiculated mass-like thickening on the contour of the urethra. It involves the anterior vaginal wall (arrow). 
The excision of all endometriotic implants is the treatment chosen to control the symptomatology. Following the histopathological response she took preoperative hormone therapy to reduce the size of the lesions and decrease the risk of postoperative incontinence.

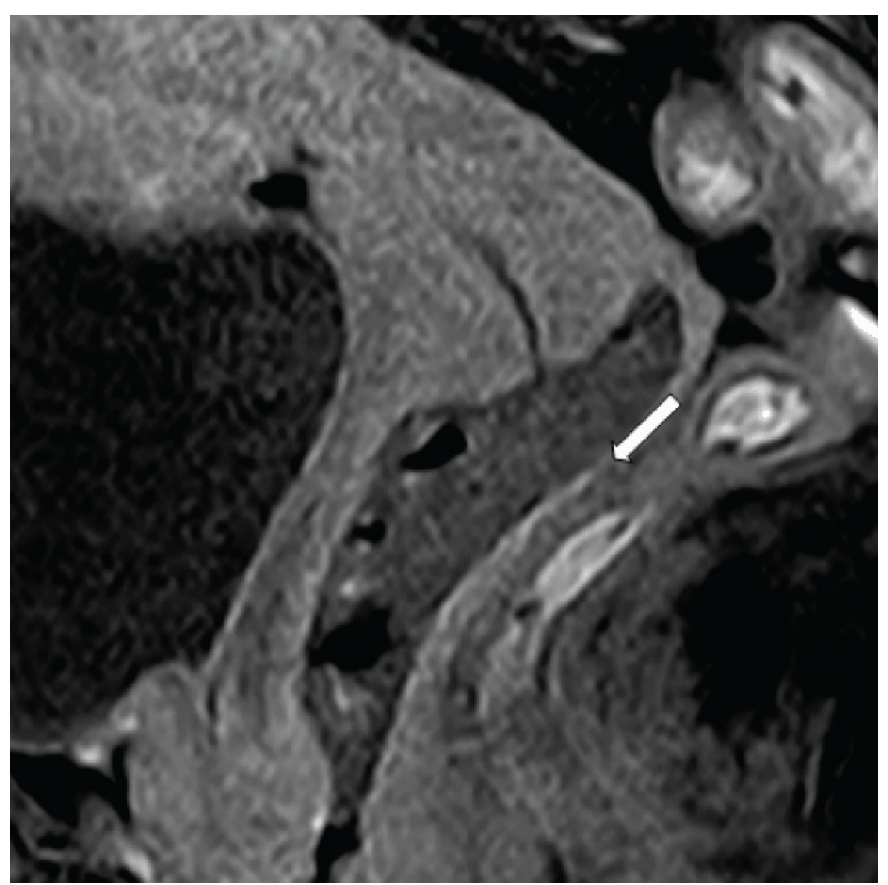

Figure 3: Sagittal T1-weighted sequence shows a thin hyperintense lines of the posterior vaginal wall indicating plaques bloody content (arrow). This finding is visible only after vaginal distension.

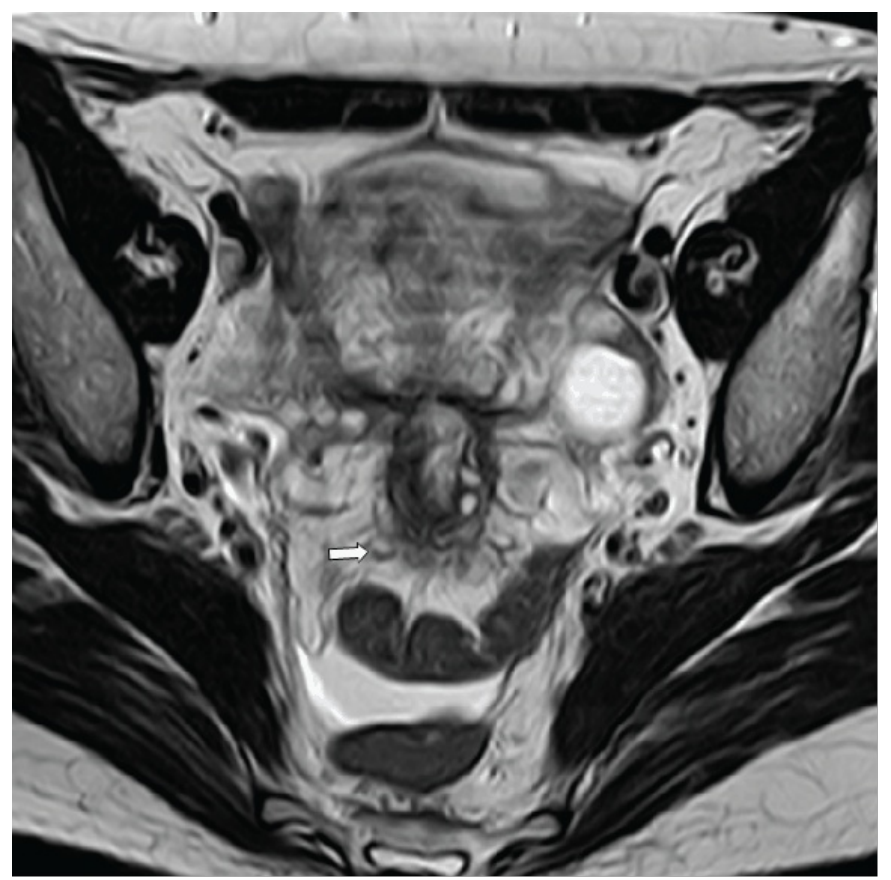

Figure 4: Axial T2-weighted sequence shows the hypointense lines located between the torus uterinus and the sigmoid related to retractile adhesions (arrow).

\section{DISCUSSION}

The pathogenesis of endometriosis remains controversial and is probably multifactorial. The most widely accepted theory is the implantion theory (Sampson 1927). According to this theory, the lesions are secondary to the implantation and the proliferation of regurgitated endometrial cells in an ectopic position. Angio- and lymphatic theory, impairment of the immune response, heritable tendencies and secreted products of endometriotic lesions may contribute to explain the pathogenesis of this disorder $[4,5]$.

In this case deep dyspareunia and pelvic pain started immediately after spontaneous interruption of pregnancy and uterine curettage. According to the implantion theory we assume that the vaginal endometriosis was the result of implantation of endometrial glands into vaginal tearing during surgical procedure of curettage. In the current literature some authors have described cases of extraperitoneal locations including vagina, RVS and mostly on episiotomy scars, after obstetrical and surgical trauma, in particular after natural delivery [6]. No author has so far described diffuse involvement of vaginal wall in presence of uterine curettage after pregnancy interruption. Our patient had a history of two cesarean sections as well.

According to a functional and clinical classification in our case the anterior, middle and posterior compartments are involved. In fact, the endometriotic lesions affect periurethral tissue (anterior compartment), vagina (middle compartment) and torus uterinus and bowel (posterior compartment) [7]. We can presume that the vaginal DIE was a previous lesion, while the others lesions arise from vaginal walls through a contigous extension. Isolated urethral involvement is poorly described in literature. It is more frequently observed as an extension from the others organs such as the bladder [7].

In this case ultrasonography was the first diagnostic tool used. It is recommended for the study of the ovary and bladder but its sensitivity is lower than that of MRI in identifying subperitoneal lesions extensions [8]. Ultrasonography reported the presence of endometrioma in the right adnexal, cyst in the left adnexal and multiple nodules of uterine fibroids.

The MRI showed a hypointense concentric thickening of the vaginal wall on T2-W images. The use of gel allowed us to better evaluate this finding. It was mainly evident in the lower third of the vagina where it caused pseudo-stenosis (Figure 1B). The pseudostenosis was not appreciable on the sequence without gel (Figure 1A).

In another recent report, we demonstrated that the use of intravaginal gel increases the sensitivity of MRI without gel in detecting DIE lesions. Diagnostic difficulties of DIE are related to the inherent features of endometriotic lesions which can have an MRI signal intensity very similar to those of the surrounding fibromuscular anatomic structures. Also DIE involves multiple closely related anatomical structures and with only subtle signal alterations. In this regard the advantage of the gel is its hypersignal on T2-W image 
which contrasts the hyposignal of the surrounding fibromuscolar anatomical structures, therefore facilitating the diagnosis [9].

Some authors claim that the presence of small foci of high signal within of fibrotic lesions can facilitate the diagnosis of DIE. Our case contradicts this assertion. In fact, the endometriotic blood lesion localized between the anterior vaginal wall and the urethra, above the perineal body, was poorly detected in the sequence without gel (Figure 1A) rather than with gel (Figure 1B). The intravaginal gel relaxing the vaginal walls and the fornices allowed a better view of the anterior deep pelvic area and of the retrocervical area, as confirmed by literature [9].

The thin lines of the posterior vaginal wall, hyperintense on $\mathrm{T} 1-\mathrm{W}$ fat saturation sequences, indicating blood plaques, was detected only after vaginal distension (Figure 3).

An MRI with endovaginal gel allows us to give essential preoperative mapping for the surgical removal of the DIE lesions. Furthermore, this method stands out because higher sensitivity compared to transvaginal ultrasonography and MRI without gel, detecting more lesions [9].

A wide excision of all endometriotic implants remains the chosen treatment and an accurate preoperative assessment of the extension of the endometriosis lesions is necessary for a successful treatment and to control the symptomatology.

In deep extraperitoneal area the radical excision of endometriosis lesions could include partial excision of the sphincter, which that may compromise the urinary continence.

In this case, hormonal therapy was used before of the surgical procedure in order to decrease the risk of postoperative incontinence and to decrease the recurrence rate. In a case series of six patients Liang et al. observed recurrence in patients treated with only surgery, while none of the patients treated with surgical and medical therapy had recurrence of endometriosis [5].

Also the use of preoperative hormonal therapy could reduce the size and facilitate the excision of the lesion reducing damage to surrounding tissue [10].

\section{CONCLUSION}

In literature, many authors have described deeply infiltrating endometriosis but none of them has so far described diffuse involvement of the vaginal wall and of the periurethral tissue without involvement of the bladder yet.

Furthermore, our study is the first one to describe a case of triple concomitant affected compartments; periurethral tissue (anterior compartment), vagina (middle compartment) and torus uterinus and bowel (posterior compartment) in patient with deep extraperitoneal endometriosis.

\section{Author Contributions}

Valeria Fiaschetti - Conception and design, Acquisition of data, Analysis and interpretation of data, Critical revision of the article, Final approval of the version to be published

Valentina Cama - Conception and design, Acquisition of data, Analysis and interpretation of data, Critical revision of the article, Final approval of the version to be published

Laura Greco - Conception and design, Acquisition of data, Analysis and interpretation of data, Critical revision of the article, Final approval of the version to be published

Maria Fornari - Conception and design, Acquisition of data, Analysis and interpretation of data, Critical revision of the article, Final approval of the version to be published

Giuseppe Sorrenti - Conception and design, Acquisition of data, Analysis and interpretation of data, Critical revision of the article, Final approval of the version to be published

Giovanni Simonetti - Conception and design, Acquisition of data, Analysis and interpretation of data, Critical revision of the article, Final approval of the version to be published

\section{Guarantor}

The corresponding author is the guarantor of submission.

\section{Conflict of Interest}

Authors declare no conflict of interest.

\section{Copyright}

(C) Valeria Fiaschetti et al. 2013; This article is distributed under the terms of Creative Commons attribution 3.0 License which permits unrestricted use, distribution and reproduction in any means provided the original authors and original publisher are properly credited. (Please see www.ijcasereportsandimages.com /copyright-policy.php for more information.)

\section{REFERENCES}

1. Viganò $\mathrm{P}$, Parazzini $\mathrm{F}$, Somigliana E, Vercellini $\mathrm{P}$. Endometriosis: epidemiology and aetiological factors. Best Pract Res Clin Obstet Gynaecol 2004 Apr;18(2):177-200.

2. Cornillie Fj, Oosterlynck D, Lauweryns JM, Koninckx PR. Deeply infiltrating pelvic endometriosis: histology and clinical significance. Fertil Steril 1990;53(6):978-83.

3. Del Frate C, Girometti R, Pittino M, et al. Deep retroperitoneal pelvic endometriosis: MR imaging appearance with laparoscopic correlation. Radiographics. 2006 Nov-Dec;26(6):1705-18.

4. Simpson JL, Bischoff FZ. Heritability and molecular genetic studies of endometriosis. Ann N Y Acad Sci 2002 Mar;955:239-51.

5. Giudice LC, Kao LC. Endometriosis. Lancet 2004 Nov 13-19;364(9447):1789-99. 
6. Odobasic A, Pasic A, Iljazovic-Latifagic E, et al. Perineal endometriosis: a case report and review of the literature. Tech Coloproctol 2010 Nov;14 Suppl 1:S25-7.

7. Coutinho A Jr, Bittencourt LK, Pires CE, et al. MR imaging in deep pelvic endometriosis: a pictorial essay. Radiographics 2011 Mar-Apr;31(2):549-67.

8. Bazot M, Darai E, Hourani R, et al. Deep pelvic endometriosis: MR imaging for diagnosis and prediction of extension of disease. Radiology 2004;232(2):379-89.

9. Fiaschetti V, Crusco S, Meschini A, et al. Deeply infiltrating endometriosis: Evaluation of retrocervical space on MRI after vaginal opacification. Eur J Radiol 2012 Nov;81(11):3638-45.

10. Chen N, Zhu L, Lang J, et al. The clinical features and management of perineal endometriosis with anal sphincter involvement: a clinical analysis of 31 cases. Hum Reprod 2012 Jun;27(6):1624-7. 